\title{
DIFFERENTIAL INEQUALITIES AND LOCAL VALENCY
}

\author{
W. K. HAYMAN
}

\begin{abstract}
An entire function $f(z)$ is said to have bounded value distribution (b.v.d.) if there exist constants $p, R$ such that the equation $f(z)=w$ never has more than $p$ roots in any disk of radius $R$. It is shown that this is the case for a particular $p$ and some $R>0$ if and only if there is a constant $C>0$ such that for all $z$
\end{abstract}

$$
\left|f^{(p+1)}(z)\right| \leqq C \max _{\nu=1 \text { to } p}\left|f^{(\nu)}(z)\right|,
$$

so that $f^{\prime}(z)$ has bounded index in the sense of Lepson.

We consider the differential equation

$$
y^{(n)}+a_{1} y^{(n-1)}+\cdots a_{n} y=0,
$$

in the disk

$$
D_{0}=\left\{z|| z-z_{0} \mid<R\right\},
$$

where $0<R \leqq \infty$ and the functions $a_{1}$ to $a_{n}$ are supposed to be regular and bounded in $D_{0}$. The solutions of (1.1) are regular in $D_{0}$ and possess there no zeros of order greater than $n-1$. This prompted Tijdeman to ask for a disk depending only on $z_{0}, R$ and the coefficients $a_{1}$ to $a_{n}$, in which the equation (1.1) is disconjugate, i.e. no solution has more than $n-1$ zeros. He later solved this problem [10] using a method due to Turan [12]. At about the same time a solution was given by Kim [5], who obtained sufficient conditions on $a_{1}$ to $a_{n}$ for (1.1) to be disconjugate in the whole of $D_{0}$.

An interesting special case occurs when $R=\infty$, so that $D_{0}$ is the open plane. In this case $a_{1}$ to $a_{n}$ must be constant and the solutions take the form

$$
y(z)=\sum_{\nu=1}^{k} p_{\nu}(z) e^{\omega_{\nu} z}
$$

where the $\omega_{\nu}$ are the roots of the characteristic equation

$$
P(\omega)=\omega^{n}+\sum_{\mu=1}^{n} a_{\mu} \omega^{n-\mu}=0 \text {. }
$$

If $\omega_{\nu}$ has multiplicity $k_{\nu}$ then $p_{\nu}(z)$ is a polynomial of degree at most $k_{\nu}-1$. With his methods Tijdeman [11] has obtained a number of striking results concerning the distribution of the zeros of (1.3). Setting 


$$
\Delta=\max _{\nu=1 \text { to } k}\left|\omega_{\nu}\right|
$$

he proved that if $N_{R}$ is the number of zeros of $y(z)$ in $D_{0}$, then

$$
N_{R} \leqq 3(n-1)+4 R \Delta \text {. }
$$

This sharpens considerably an earlier result of Dancs and Turán [2]. He also proved [11, Theorem 2]

THEOREM A. If $R \Delta \leqq \min \left\{n^{-2},(24)^{-2}\right\}$, then $N_{R} \leqq n-1$, so that (1.1) is disconjugate in $D_{0}$.

We can clearly consider $y(z)-w$ instead of $w$, where $w$ is a constant, at the expense of replacing the order $n$ of (1.1) by $n+1$. Thus we deduce at once from Tijdeman's results

THEOREM B. If $N_{R}(w)$ denotes the number of roots of $y(z)=w$ in $D_{0}$, then

$$
N_{R}(w) \leqq 3 n+4 R \Delta,
$$

and if $R \Delta \leqq \min \left\{(n+1)^{-2},(24)^{-2}\right\}$, we have

$$
N_{R}(w) \leqq n
$$

The bounds in (1.5) and (1.6) are independent of $w$ and $z_{0}$. We can restate (1.6) by saying that $y(z)$ is at most $n$-valent in any disk of radius $R$ in the open plane.

We now quote the main result of $\operatorname{Kim}[5, \mathrm{pp} .721,722]$, which may be stated in the following form

THEOREM C. Suppose that $y(z)$ is regular in $D_{0}$, and has $n$ zeros there. Then there exists $z_{1}$ in $D_{0}$ such that

$$
\begin{gathered}
\left|y\left(z_{1}\right)\right|<\frac{1}{n !}\left(R-\left|z_{1}-z_{0}\right|\right)\left(R+\left|z_{1}-z_{0}\right|\right)^{n-1}\left|y^{(n)}\left(z_{1}\right)\right|, \\
\left|y^{(n-k)}\left(z_{1}\right)\right|<\frac{1}{k !}\left(R+\left|z_{1}-z_{0}\right|\right)^{k}\left|y^{(n)}\left(z_{1}\right)\right|, 1 \leqq k \leqq n-1 .
\end{gathered}
$$

Hence if

$$
\sum_{k=1}^{n-1} \frac{\left(R+\left|z-z_{0}\right|\right)^{k}}{k !}\left|a_{k}(z)\right|+\frac{\left(R-\left|z-z_{0}\right| \mid\right)\left(R+\left|z-z_{0}\right|\right)^{n-1}}{n !}\left|a_{n}(z)\right| \leqq 1
$$

in $D_{0}$ then (1.1) is disconjugate in $D_{0}$.

Both Kim's and Tijdeman's results can also be applied to equations 
(1.1) with polynomial coefficients.

In connection with Theorem B, Turán [3, problem 2.28] considered functions $y(z)$ which satisfy (1.6) for fixed constants $R$ and $n$ and every $z_{0}$. He called such $y(z)$ functions of bounded value distribution (b.v.d.). Suppose that in the equation (1.1) the coefficients are integral functions, and that every solution $y(z)$ has b.v.d.. Then he asked if the $a_{1}$ to $a_{n}$ are necessarily constants. This was proved to be the case by Wittich [13] even under the weaker hypothesis, that for every solution $y(z)$ there exists at least one $w \neq 0$ and a constant $R$, such that $N_{R}(w)$ is bounded above for all $z_{0}$. Turán [3, problem 2.28] also asked whether a b.v.d. function necessarily has at most mean type of order one in the plane.

A related question was raised by Lepson [6]. Let $f(z)$ be an entire function and for each $z$ let $N(z)$ be the least integer such that

$$
\sup _{0 \leqq j<\infty}\left|\frac{f^{(j)}(z)}{j !}\right|=\frac{\left|f^{(N)}(z)\right|}{N !} .
$$

If $N(z)$ is bounded above for varying $z$, then $f(z)$ is said to be of bounded index, and the least upper bound $N$ of $N(z)$ is called the index of $f(z)$. It was shown by Shah [7] that the solutions of equations (1.1) have bounded index if the $a_{n}(z)$ are constants. More generally if the $a_{n}(z)$ are rational functions which remain bounded at $\infty$, then any solution of (1.1) which is an entire function has bounded index [8]. Shah also showed that any function of bounded index has order 1 , type $N+1$ at most [7]. This result is sharp as shown by $f(z)=\exp \{(N+1) z\}$. It is evident that if $f^{\prime}(z)$ has bounded index $N$, then $f(z)$ has bounded index at most $N+1$. The converse is however false, as an example at the end of the paper will show. Another example has just been given by Shah [9].

Lepson in conversation with me raised the question as to whether the functions of bounded index and b.v.d. were related. This problem was the basis of the present paper. We can settle the question in one direction very simply by quoting the following form of a classical result on $p$-valent functions. We denote as usual positive absolute constants by $A$, constants depending on $p, q$ etc. by $A(p), A(p, q)$, particular constants by $A_{1}, A_{2}, \cdots$ etc.

THEOREM D. Suppose that $f(z)$ is p-valent in $D_{0}$, i.e. that $f(z)$ is regular in $D_{0}$ and assumes no value more than $p$ times there; then for $j>p$

$$
\frac{\left|f^{(j)}\left(z_{0}\right)\right| R^{j}}{j !} \leqq A_{1}(p) j^{2 p} \max _{\nu=1 \text { to } p} \frac{\left|f^{(\nu)}\left(z_{0}\right)\right| R^{\nu}}{\nu !}
$$


The result (with $j^{2 p-1}$ instead of $j^{2 p}$ ) is due to Biernacki [1]. The best known numerical value of $A_{1}(p)$ is due to Jenkins and Oikawa [4], who proved a bound for the maximum modulus of $p$-valent functions from which Theorem $\mathrm{D}$ follows with $A_{1}(p)=\left(A_{1} / p\right)^{2 p}$. If $R \geqq 2$ we shall deduce from (1.11) that $f^{\prime}(z)$ has index at most $A_{3} p$. If $R<2$, simple geometrical considerations show that $f(z)$ is at most $p_{1}$-valent in $\left|z-z_{0}\right|<2$, for every $z_{0}$, where $p_{1}<A_{4} p / R^{2}$. Thus in this case $f(z)$ has index at most $A_{5} p / R^{2}$. Thus we have the following

THeorem 1. If $f(z)$ is p-valent in $\left|z-z_{0}\right|<R$, for every $z_{0}$ and a fixed $R$, then $f^{\prime}(z)$ has index at most $A p \max \left(1, R^{-2}\right)$. If $R \geqq A_{6}^{p}$, then $f^{\prime}(z)$ has index at most $p-1$. In particular if $f(z)$ has b.v.d. then $f^{\prime}(z)$ has bounded index.

2. Statement of further results. We shall in this paper prove a local converse to Theorem $\mathrm{D}$, i.e. we shall show that if $f(z)$ satisfies an inequality such as (1.11) in a disk, then $f(z)$ is $p$-valent in a suitable smaller disk. The result will also enable us to improve the Theorems of Tijdeman and Kim in certain cases. It is convenient to define

$$
f_{n}(z)=\max _{0 \leqq \nu \leqq n-1} \mid f^{(\nu)}(z) .
$$

Our first result is

Theorem 2. If $f(z)$ is regular in $D_{0}$ and satisfies

$$
\left|f^{(n)}(z)\right| \leqq f_{n}(z)
$$

there, then we have in $D_{0}$

$$
f_{n}(z) \leqq f_{n}\left(z_{0}\right) \exp \left(\left|z-z_{0}\right|\right) .
$$

CoRollary 1. If $f(z)$ is an integral function satisfying (2.2) in the whole open plane, then

$$
|f(z)| \leqq f_{n}(0) e^{|z|}
$$

there, so that $f(z)$ has at most order 1, type one.

Corollary 2. If $f(z)$ is an integral function of bounded index $N$, then

1 It will follow at once from (2.8), that this estimate can be replaced by $A p \max \left(1, R^{-1}\right)$. 


$$
|f(z)| \leqq e^{(N+1)|z|} \max _{0 \leqq \nu \leqq N} \frac{\left|f^{(\nu)}(0)\right|}{(N+1)^{\nu}} \leqq f_{N+1}(0) e^{(N+1)|z|}
$$

The Corollary 2 sharpens the theorem of Shah [7] previously referred to. The function $f(z)=e^{(N+1) z}$, which has index $N$, shows that (2.5) cannot be further sharpened except possibly for the constant $f_{N+1}(0)$. On the other hand the inequality (2.3) is sharp, as $f(z)=e^{z}$ shows. This is the reason why (2.2) is more convenient as a normalisation than (1.10) for the purpose of this paper. Our main result is an analogue of Theorem $\mathrm{C}$. This is

THEOREM 3. Suppose that $f(z)$ is regular in $|z|<2 n$ and satisfies (2.2) there. Then $f(z)$ possesses at most $n-1$ zeros in

$$
|z| \leqq n^{1 / 2} /(e \sqrt{20})<n^{1 ! 2} / 12.2 \text {. }
$$

Let us compare this result with the conclusion to be drawn from Theorem $\mathrm{C}$.

THEOREM E. Suppose that $f(z)$ is regular and satisfies (2.2) in $|z| \leqq R$, where $R \leqq 1 / 2$. Then $f(z)$ has at most $n-1$ zeros there.

Theorem $\mathrm{E}$ is stronger than Theorem 3 for $n \leqq 37$, and we only have to assume that $f(z)$ satisfies the hypotheses in $|z| \leqq R$. On the other hand for $n \geqq 38$, Theorem 3 yieds a larger disk in which there are at most $n-1$ zeros, at the cost of assuming that $f(z)$ satisfies the hypotheses in a still larger disk.

The order of magnitude in Theorem 3 is the correct one for large $n$. We set

$$
\begin{gathered}
f(z)=\frac{1}{n !}\left(z^{2}-3(n-1)\right)^{(1 / 2) n}, \quad \text { if } n \text { is even } ; \\
f(z)=\frac{1}{n !} z\left(z^{2}-3 n\right)^{(1 / 2)(n-1)}, \text { if } n \text { is odd } .
\end{gathered}
$$

Then $f(z)$ has $n$ zeros in $|z| \leqq \sqrt{(3 n)}$. On the other hand

$$
f^{(n)}(z)=1, \quad f^{(n-1)}(z)=z, \quad f^{(n-2)}(z)=\frac{1}{2} z^{2}-\frac{3}{2},
$$

so that we have for all $z$

$$
1=\left|f^{(n)}(z)\right| \leqq \max \left(\left|f^{(n-1)}(z)\right|,\left|f^{(n-2}(z)\right|\right) \leqq f_{n}(z) \text {. }
$$

Thus we cannot replace $n^{1 / 2} /(12.2)$, by $n^{1 / 2} \sqrt{3}$ in Theorem 3 , even if (2.2) is satisfied in the whole plane. 
By applying Theorem 3 to $f(z)-w$, we obtain the desired converse to Theorem D. This is

THEOREM 4. Suppose that $f(z)$ is regular in $D_{0}$ and satisfies there

$$
(C R)^{p+1}\left|\frac{f^{(p+1)}(z)}{(p+1) !}\right| \leqq \max _{1 \leqq \nu \leqq p}(C R)^{\nu} \frac{\left|f^{(\nu)}(z)\right|}{\nu !},
$$

with $C \leqq 1 / 2$. Then $f(z)$ is $p$-valent in $\left|z-z_{0}\right| \leqq C R /\left\{12 \cdot 2(p+1)^{1 / 2}\right\}$.

COROLlaRy. An integral function $f(z)$ has b.v.d. if and only if $f^{\prime}(z)$ has bounded index. More particularly if $p(R)$ is the upper bound of the valencies of $f(z)$ in $\left|z-z_{0}\right|<R$, for varying $z_{0}$, and $N$ is the index of $f^{\prime}(z)$ we have

$$
p\left\{\frac{1}{12 \cdot 2}(N+2)^{-1 / 2}\right\} \leqq N+1 \leqq p\left(A_{6}^{N}\right)
$$

We can also prove

THEOREM 5. Suppose that $f^{\prime}(z)$ is a function of bounded index $N$ and let $p(R)$ be defined as above. Then

$$
A_{7}(N+1) \leqq p(1) \leqq A_{8}(N+1)
$$

Furthermore for $R \geqq 1$, we have

$$
p(R)<(N+1) e(R+2) .
$$

Corollary. If $0<R_{1}<R_{2}<\infty$ then we have

$$
\frac{p\left(R_{2}\right)}{R_{2}}<A_{9} \frac{p\left(R_{1}\right)}{R_{1}}
$$

We now turn to applications to the disconjugacy problem of the equation (1.1). We write

$$
\alpha_{\nu}=\sup _{z \in D_{0}} a_{\nu}(z) \mid, \alpha_{0}=\sup _{1 \leqq \nu \leqq n} \alpha_{\nu}^{1 / \nu}
$$

and let $t_{0}$ be the positive root of the equation

$$
\sum_{\nu=1}^{n} \alpha_{\nu} t_{0}^{\nu}=1
$$

Then we have evidently

$$
\frac{1}{2 \alpha_{0}}<t_{0} \leqq \frac{1}{\alpha_{0}}
$$


We suppose that $y(z)$ is a solution of (1.1) and set $f(z)=y\left(z_{0}+t z\right)$ where $t \leqq t_{0}$. Then

$$
f^{(\nu)}(z)=t^{\nu} y^{\nu}\left(z_{0}+t z\right), \quad \nu=0 \text { to } n,
$$

so that $f(z)$ satisfies for $|z| \leqq R / t$ the differential equation

$$
f^{(n)}(z)=-\sum_{1}^{n} a_{\nu}\left(z_{0}+t z\right) t^{\nu} f^{(n-\nu)}(t z) .
$$

In view of (2.9), (2.10) this leads to (2.2). Thus by applying Theorem 3 to $f(z)$, with $t=t_{1}=\min \left\{t_{0}, R / 2 n\right\}$ we obtain

THEOREM 6. If $y(z)$ is a solution of the equation (1.1) in $D_{0}$, then $y(z)$ has at most $n-1$ zeros in

$$
\left|z-z_{0}\right| \leqq R_{1}=\min \left\{t_{0} n^{1 / 2} /(e \sqrt{20}), R /\left[4 e(5 n)^{1 / 2}\right]\right\}
$$

i.e. the equation is disconjugate in $\left|z-z_{0}\right|<R_{1}$.

To compare Theorem $C$ and Theorem 6, we take $\left|a_{k}\right|$ constant in (1.1) and $R_{1}=1$. Then the condition for Theorem $\mathrm{C}$ is certainly satisfied if

$$
\sum_{k=1}^{n} \frac{2^{k}\left|a_{k}\right|}{k !} \leqq 1,
$$

while the condition for Theorem 6 is that

$$
\sum_{k=1}^{n}\left|a_{k}\right| \frac{(12.2)^{k}}{n^{(1 / 2) k}} \leqq 1 .
$$

Thus Kim's condition is weaker and so his Theorem is stronger unless $n$ is very large, and the $a_{k}$ for small $k$ relatively large compared with the others, when Theorem 6 gives a better result. prove

Finally we return to the exponential polynomials (1.3). We shall

THEOREM 7. If $y(z)$ is the exponential polynomial (1.3) then $y(z)$ has at most $n-1$ zeros in $\left|z-z_{0}\right|<\Delta^{-1} \max \left\{0.025,0.15 n^{-1 / 2}\right\}$.

The result with $n>36$ will be deduced from Therem 6 and when $n \leqq 36$ from Theorem E. Both the results are somewhat sharper than the conclusion of Tijdeman's Theorem A.

3. Proof of Theorem 2. Suppose that $f(z)$ is regular in $D_{0}$ and satisfies (2.2) there. We set for a fixed real $\theta$

$$
g(t)=\max _{0 \leqq \nu \leqq n-1}\left|f^{(\nu)}\left(z_{0}+t e^{i \theta}\right)\right|, \quad 0 \leqq t<R .
$$


The interval $[0, R)$ can be divided into a finite number of subintervals in which one of the derivatives $\left|f^{(\nu)}\left(z_{0}+t e^{i \theta}\right)\right|$ is maximal. Thus $g(t)$ is continuous and except for a finite number of points we have in $(0, R)$ for some $\nu, 0 \leqq \nu \leqq n-1$

$$
g^{\prime}(t) \leqq\left|f^{(\nu+1)}\left(z+t e^{i \theta}\right)\right| \leqq g(t)
$$

in view of (2.2). Thus $e^{-t} g(t)$ is nonincreasing in $[0, R)$ and this yields (2.3), when we set $z=z_{0}+t e^{i \theta}$. We deduce (2.4), on setting $z_{0}=0$.

Finally to deduce Corollary 2, we suppose that $f(z)$ has index bounded by $N$ in the whole plane, so that in view of (1.10) we have

$$
\frac{\left|f^{(N+1}(z)\right|}{(N+1) !} \leqq \max _{0 \leqq \nu \leqq N} \frac{\left|f^{(\nu)}(z)\right|}{\nu !} .
$$

We set $g(z)=f(t z)$, where $t=(N+1)^{-1}$, and deduce that

$$
\left|g^{(N+1)}(z)\right| \leqq \max _{0 \leqq \nu \leqq N} \frac{t^{(N+1-\nu)}(N+1) !}{\nu !}\left|g^{(\nu)}(z)\right| \leqq \max _{0 \leqq \nu \leqq N}\left|g^{(\nu)}(z)\right| \text {. }
$$

Thus $g(z)$ satisfies (2.2), with $n=N+1$, and we deduce from (2.4) that

$$
|g(z)| \leqq g_{N+1}(0) e^{|z|}=\max _{0 \leqq \nu . N}\left|g^{(\nu)}(0)\right| e^{|z|}=\max _{0 \leqq \nu \leq N} t^{\nu}\left|f^{(\nu)}(0)\right| e^{|z|} \leqq f_{N+1}(0) e^{|z|} .
$$

Since $f(z)=g[(N+1) z]$, we deduce (2.5).

4. Proof of Theorem 3. To prove Theorem 3 we need, apart from Theorem 2, two subsidiary results.

Lemma 1. Let $z_{\nu}, \nu=1$ to $n$ be complex numbers such that $\max _{1 \leqq \nu \geqq n}\left|z_{\nu}\right|=\rho_{0}$. If further

$$
\phi(z)=\left\{\prod_{\nu=1}^{n}\left(1-z_{\nu} z\right)\right\}^{\varepsilon}=\sum_{0}^{\infty} b_{k} z^{k},
$$

where $b_{1}=0$, and $\varepsilon=1$ or -1 , then

$$
\left|b_{k}\right|<n^{(1 \mid 2) k} \rho_{0}^{k}, k>1 .
$$

The Lemma is false, without the hypothesis $b_{1}=0$. Thus if $\phi(z)=(z+1)^{n}$, then

$$
\rho_{0}=1, b_{k}=n(n-1) \cdots(n-k+1) / k ! \sim \frac{n^{k}}{k !},
$$

as $n \rightarrow \infty$ for fixed $k$.

With the hypotheses of Lemma 1 , we can set 


$$
\psi(z)=\sum_{\nu=1}^{n} \log \left(\frac{1}{1-z_{\nu} z}\right)=\sum_{k=1}^{\infty} \frac{S_{k} z^{k}}{k}
$$

where $S_{1}=0$ and

$$
\left|S_{k}\right|=\left|\sum_{\nu=1}^{n} z_{\nu}^{k}\right| \leqq n \rho_{0}^{k} \leqq\left(n^{1 / 2} \rho_{0}\right)^{k}, \quad k \geqq 2 .
$$

Since $\phi(z)=\exp \{\mp \psi(z)\}$, we deduce that the coefficents of $\phi(z)$ cannot exceed those of $\exp \left\{\sum_{1}^{\infty}\left(\left|S_{k}\right| / k\right) z^{k}\right\}$ which are smaller than those of

$$
\exp \left\{\sum_{1}^{\infty} \frac{\left(n^{1 / 2} \rho_{0} z\right)^{k}}{k}\right\}=\left(1-n^{1 / 2} \rho_{0} z\right)^{-1}
$$

This prove Lemma 1.

We have next

LEMMA 2. Suppose that $g(z)=\sum_{0}^{\infty} g_{\nu} z^{\nu}$ is regular in $|z| \leqq \rho$, and that

$$
\sum_{\nu=n}^{\infty}\left|g_{\nu}\right|^{2} \rho^{2 \nu} \leqq \sum_{\nu=0}^{n-1}\left|g_{\nu}\right|^{2} \rho^{2 \nu}
$$

Further let $z_{\nu}, 1 \leqq \nu \leqq n$ be zeros of $g(z)$ in $|z| \leqq \rho$, such that

$$
\sum_{\nu=1}^{n} z_{\nu}=0
$$

Here multiple zeros may be counted according to their multiplicity. Then

$$
\rho_{0}=\max _{1 \leqq \nu \leqq n}\left|z_{\nu}\right|>\rho(5 n)^{-1 / 2} .
$$

Here also the condition (4.2) is essential. For without this we may take

$$
g(z)=(z+a)^{n}
$$

which has $n$ zeros at $z=-a$, and certainly satisfies (4.1) if

$$
\rho^{2 n} \leqq n^{2}|a|^{2} \rho^{2 n-2} \text {, i.e. } \rho \leqq n|a| \text {. }
$$

Thus without (4.2) we can certainly not assert $\rho_{0}>\rho / n$ instead of (4.3).

To prove (4.3) we assume without loss of generality that $\rho=1$, since otherwise we may consider $g(\rho z)$ instead of $g(z)$. We now set

$$
h(z)=\prod_{\nu=1}^{n} \frac{z-z_{\nu}}{1-z_{\nu} z}=\sum_{0}^{\infty} h_{\nu} z^{\nu}
$$

It follows from Lemma 1 that 


$$
\prod_{\nu=1}^{n} \frac{1}{1-z_{\nu} z}=\sum_{0}^{\infty} b_{k} z^{k}, \quad \text { where } \quad b_{1}=0,\left|b_{k}\right|<\left(\rho_{0} n^{1 / 2}\right)^{k}, k>1 .
$$

Similarly if

$$
\prod_{\nu=1}^{n}\left(z-z_{\nu}\right)=\sum_{k=0}^{n} c_{k} z^{n-k}
$$

then

$$
\prod_{\nu=1}^{n}\left(1-\frac{z_{\nu}}{z}\right)=\sum_{0}^{n} c_{k} z^{-k}, \quad \prod_{\nu=1}^{n}\left(1-z_{\nu} z\right)=\sum_{k=0}^{n} c_{k} z^{k},
$$

and now Lemma 1 shows that

$$
c_{1}=0,\left|c_{k}\right|<\left(\rho_{0} n^{1 / 2}\right)^{k}, \quad k \geqq 2 .
$$

We set $\rho_{0} n^{1 / 2}=t,\left(1-t^{2}\right)^{-1}=\tau$. Thus we have for $k \geqq 1$

$$
\left|h_{n-k}\right|=\left|\sum_{p=k}^{n} c_{p} b_{p-k}\right|<\sum_{p=k}^{n} t^{2 p-k}<\frac{t^{k}}{1-t^{2}}=\tau t^{k} .
$$

We now set

$$
g(z)=G(z) h(z),
$$

and assume that $\rho_{0}<\rho$, since otherwise there is nothing to prove. Then $G(z)$ is regular in $|z| \leqq 1$, and $|G(z)|=|g(z)|$ for $|z|=1$. Thus if

$$
G(z)=\sum_{\nu=0}^{\infty} G_{\nu} z^{\nu}
$$

we have

$$
\sum_{\nu=0}^{\infty}\left|G_{\nu}\right|^{2}=\sum_{0}^{\infty}\left|g_{\nu}\right|^{2}=\sigma
$$

say. Now for $p<n$, we have

$$
\begin{aligned}
\left|g_{p}\right|^{2} & =\left|\sum_{k=0}^{p} G_{k} h_{p-k}\right|^{2} \leqq \sum_{k=0}^{p}\left|G_{k}\right|^{2} \sum_{k=0}^{p}\left|h_{p-k}\right|^{2} \\
& <\sigma \sum_{k=0}^{p} \tau^{2} t^{2(n+k-p)}<\frac{\sigma \tau^{2} t^{2 n-2 p}}{1-t^{2}}=\sigma \tau^{3} t^{2 n-2 p} .
\end{aligned}
$$

This yields in turn

$$
\sum_{p=0}^{n-1}\left|g_{p}\right|^{2}<\sigma \tau^{3} \sum_{p=0}^{n-1} t^{2 n-2 p}<\sigma \tau^{4} t^{2}<\frac{1}{2} \sigma,
$$

if $t^{2} \leqq 1 / 5$, so that $\tau \leqq 5 / 4$. This contradicts (4.1). Thus $t>5^{-1 / 2}$, and Lemma 2 is proved. 
4.1. We can now complete the proof of Theorem 3. We assume that $f(z)$ has $n$ zeros, $z_{1}$ to $z_{n}$, in $|z| \leqq \rho_{0}$, and set

$$
\begin{gathered}
z_{0}=\frac{1}{n} \sum_{\nu=1}^{n} z_{\nu}, \quad z_{\nu}^{\prime}=z_{\nu}+z_{0}, \\
F(z)=f\left(z_{0}+z\right) .
\end{gathered}
$$

Then $F(z)$ has the $n$ zeros $z_{\nu}^{\prime}$ with $\left|z_{\nu}^{\prime}\right| \leqq 2 \rho_{0}$, and $\sum z_{\nu}^{\prime}=0$. Further $F(z)$ satisfies (2.2) in $|z|<2 n-\rho_{0}$. From this we proceed to obtain a lower bound for $\rho_{0}$. We assume $\rho_{0}<(1 / 2) n$, since otherwise there is nothing to prove, set

$$
\mu=F_{n}(0)=\max _{0 \leqq \nu \leqq n-1}\left|F^{(\nu)}(0)\right|,
$$

and write

$$
F(z)=\sum_{0}^{\infty} F_{\nu} z^{\nu}
$$

Then it follows from (2.3) that

$$
M_{n}(R)=\max _{|z| \leqq R}\left|F^{(n)}(z)\right| \leqq \mu e^{R}, \quad 0 \leqq R \leqq n .
$$

Further

$$
F^{(n)}(z)=\sum_{\nu=n}^{\infty} \frac{\nu !}{(\nu-n) !} F_{\nu} z^{\nu-n}
$$

Hence Cauchy's inequality yields for $0<R<n$

$$
\frac{\nu !}{(\nu-n) !}\left|F_{\nu}\right| \leqq \frac{M_{n}(R)}{R^{\nu-n}} \leqq \frac{\mu e^{R}}{R^{\nu-n}}, \quad \nu \geqq n .
$$

We now set $\nu=n+t, R=\inf (t, n-1)$ in (4.4) and take $\rho=n / e$ in Lemma 2. Then (4.4) may be written as

$$
\left|F_{n+t}\right| \rho^{n+t} \leqq \frac{\mu \rho^{n}}{n !} \frac{n ! t !}{(n+t) !}\left(\frac{\rho}{R}\right)^{t} e^{R}=\frac{\mu \rho^{n}}{n !} U_{t}
$$

say, where

$$
\begin{aligned}
& U_{0}=1 \\
& U_{t}=\frac{n ! t !}{(n+t) !}\left(\frac{n}{e}\right)^{t}\left(\frac{e}{t}\right)^{t}, \quad 1 \leqq t \leqq n-1 \\
& U_{t}=\frac{n ! t !}{(n+t) !} \cdot\left(\frac{n}{e}\right)^{t} \frac{e^{n-1}}{(n-1)^{t}}, \quad t \geqq n .
\end{aligned}
$$

We proceed to prove that 


$$
U_{t} \leqq t^{-1}, \quad t \geqq 1
$$

Suppose first that $1 \leqq t \leqq n-1$. Then

$$
U_{t}=\frac{n ! n^{t}}{(n+t) !} \cdot \frac{t !}{t^{t}} \leqq \frac{1}{t} \cdot \frac{2}{t} \cdots \frac{t}{t} \leqq \frac{1}{t}
$$

as required. Next for $n=2, t>1$ we have

$$
U_{t}=\frac{2}{(t+1)(t+2)} e\left(\frac{2}{e}\right)^{t} \leqq\left(\frac{2}{e}\right)^{t-1} \frac{1}{t+1}<\frac{1}{t} .
$$

If $n>2, t=n$, we have

$$
\begin{aligned}
U_{t} & =\left(1+\frac{1}{n-1}\right)^{n} e^{-1} \frac{(n !)^{2}}{(2 n) !}<\frac{n}{n-1} \frac{1 \cdot 2 \cdots n}{(n+1)(n+2) \cdots 2 n} \\
& \leqq \frac{n}{n-1} \frac{1 \cdot 2}{2 n(2 n-1)}<\frac{1}{2 n-1}<\frac{1}{t} .
\end{aligned}
$$

Finally if $n>2, t>n$, then

$$
\begin{aligned}
U_{t} & =\frac{n ! t !}{(n+t) !}\left(\frac{n}{n-1}\right)^{t} e^{n-1-t} \\
& \leqq \frac{n ! t !}{(n+t) !} e^{t /(n-1)+n-1-t}=\frac{n ! t !}{(n+t) !} \exp \left\{n-1-t \frac{n-2}{n-1}\right\} \\
& \leqq \frac{n ! t !}{(n+t) !} \leqq \frac{1}{n+t}<\frac{1}{t} .
\end{aligned}
$$

Thus (4.6) holds in all cases.

We deduce from (4.5) and (4.6) that

$$
\begin{aligned}
\sum_{t=0}^{\infty}\left|F_{n+t}\right|^{2} \rho^{2 n+2 t} & \leqq \frac{\mu^{2} \rho^{2 n}}{(n !)^{2}} \sum_{t=0}^{\infty} U_{t}^{2} \\
& \leqq \frac{\mu^{2} \rho^{2 n}}{(n !)^{2}}\left(1+\sum_{t=1}^{\infty} t^{-2}\right)=\frac{\mu^{2} \rho^{2 n-2}}{((n-1) !)^{2}} \cdot \frac{\rho^{2}}{n^{2}}\left(1+\frac{\pi^{2}}{6}\right) \\
& =e^{-2}\left(1+\frac{\pi^{2}}{6}\right) \frac{\mu^{2} \rho^{2 n-2}}{((n-1) !)^{2}}<\left\{\frac{\mu \rho^{n-1}}{(n-1) !}\right\}^{2} .
\end{aligned}
$$

On the other hand

$$
\begin{aligned}
\sum_{\nu=0}^{n-1}\left|F_{\nu}\right|^{2} \rho^{2 \nu} & \geqq \max _{0 \leqq \nu \leqq n-1}\left|F_{\nu}\right|^{2} \rho^{2 \nu} \\
& =\max _{0 \leqq \nu \leqq n-1}\left(\nu !\left|F_{\nu}\right|\right)^{2} \frac{\rho^{2 \nu}}{(\nu !)^{2}} \geqq \max _{0 \leqq \nu \leqq n-1}\left(\nu !\left|F_{\nu}\right|\right)^{2} \inf _{0 \leqq \nu \leqq n-1} \frac{\rho^{2 \nu}}{(\nu !)^{2}} \cdot \\
& =\frac{\mu^{2} \rho^{2 n-2}}{((n-1) !)^{2}} .
\end{aligned}
$$

In fact for $\rho>1, \rho^{\nu} / \nu$ ! first increases and then decreases, as $\nu$ 
increases from $\nu=0$, and for $\rho \leqq 1, \rho^{\nu} / \nu$ ! is steadily decreasing in this range. In either case $\rho^{\nu} / \nu$ ! attains its minimum in the range $0 \leqq \nu \leqq n-1$ either at $\nu=0$ or $\nu=n-1$. Since

$$
\frac{\rho^{n-1}}{(n-1) !}=\left(\frac{n}{e}\right)^{n-1} \frac{1}{(n-1) !}
$$

decreases with increasing $n$ and is equal to 1 for $n=1$, we deduce that the minimum of $\rho^{\nu} / \nu$ ! is attained at $\nu=n-1$, and (4.8) follows.

Combining (4.7) and (4.8) we deduce that

$$
\sum_{0}^{n-1}\left|F_{\nu}\right|^{2} \rho^{2 \nu}>\sum_{\nu=n}^{\infty}\left|F_{\nu}\right|^{2} \rho^{2 \nu}
$$

Thus we can apply Lemma 2. Since $F(z)$ has the $n$ zeros $z_{\nu}^{\prime}$ with $\left|z_{\nu}^{\prime}\right| \leqq 2 \rho_{0}$ and $\sum z_{\nu}^{\prime}=0$ we deduce from Lemma 2 that

$$
2 \rho_{0}>\rho(5 n)^{-1 / 2}=n^{1 / 2} e^{-1} 5^{-1 / 2}, \rho_{0}>n^{1 / 2} /\left(20^{1 / 2} e\right) .
$$

This proves Theorem 3 .

5. Exponential polynomials. We have already seen how to deduce Theorem 6 from Theorem 3. We proceed now to deduce Theorem 7. Let

$$
y(z)=\sum_{\nu=1}^{k} p_{\nu}(z) e^{\omega_{\nu}(z)}
$$

be an exponential polynomial (1.3), and set

$$
\omega_{0}=\frac{1}{n} \sum_{1}^{n} \omega_{\nu}, \omega_{\nu}^{\prime}=\omega_{\nu}-\omega_{0},
$$

where each $\omega_{\nu}$ is counted with multiplicity $k_{\nu}$, and $k_{\nu}-1$ is the degree of $p_{\nu}(z)$. Then $f(z)=y(z) e^{-\omega_{0} z}$ satisfies the differential equation

$$
P(D) f=0,
$$

where

$$
P(D)=\prod_{\nu=1}^{n}\left(D-\omega_{\nu}^{\prime}\right)=D^{n}+b_{1} D^{n-1}+\cdots b_{n}
$$

say. Also if

$$
\Delta=\max _{1 \leqq \nu \geqq n}\left|\omega_{\nu}\right|, \text { then } \Delta^{\prime}=\max _{1 \leqq \nu \leqq n}\left|\omega_{\nu}^{\prime}\right| \leqq 2 \Delta
$$

and since $\Sigma \omega_{\nu}^{\prime}=0$, we deduce from Lemma 1 that

$$
\left|b_{k}\right| \leqq \Delta^{\prime k} n^{(1 / 2) k}, \quad k \geqq 2 ; b_{1}=0 .
$$


We choose

$$
t_{0}=\alpha /\left(2 \Delta n^{1 / 2}\right), \text { where } \alpha=\frac{\sqrt{5-1}}{2}, \text { so that } \alpha^{2}+\alpha=1,
$$

and consider $g(z)=f\left(t_{0} z\right)$. Then $g(z)$ satisfies differential equation

$$
\left(D^{n}+\sum_{\nu:=2}^{n} t_{0}^{\nu} b_{\nu} D^{n-\nu}\right) g(z)=0,
$$

and

$$
\sum_{2}^{n} t_{0}^{\nu}\left|b_{\nu}\right| \leqq \sum_{2}^{n}\left(2 t_{0} \Delta n^{1 / 2}\right)^{k}<\sum_{2}^{\infty} \alpha^{k}=\frac{\alpha^{2}}{1-\alpha}=1
$$

It now follows from Theorem 6 that $f(z)$ and so $y(z)$ has at most $n-1$ zeros in

$$
|z| \leqq n^{1 / 2} t_{0} / e \sqrt{20}=\alpha /(4 e \sqrt{5 \Delta}) \doteqdot(39 \cdot 3 \Delta)^{-1}
$$

This proves one of the inequalities of Theorem 7 .

To obtain the other we first note that Theorem $\mathrm{E}$ follows at once from Theorem C. For if $f(z)$ has $n$ zeros in $|z|<R$, where $R \leqq 1 / 2$, let $z_{1}$ be a point such that (1.7), (1.8) hold with $f(z)$ instead of $y(z)$. Then

$$
\left|f^{(n-k)}\left(z_{1}\right)\right|<\frac{(2 R)^{k}}{k !}\left|f^{(n)}\left(z_{1}\right)\right| \leqq\left|f^{(n)}\left(z_{1}\right)\right|, \quad 1 \leqq k \leqq n
$$

and this contradicts (2.2). Thus Theorem $\mathrm{E}$ is proved. It follows from (5.1) that $g(z)$ satisfies (2.2) and so has at most $n-1$ zeros in $|z|<1 / 2$, so that $f(z)$ has at most $n-1$ zeros in $|z|<(1 / 2) t_{0}$. This completes the proof of Theorem 7 .

6. Index and local valency. It remains to prove Theorems 1,4 and 5 and to this we now turn. We start by applying Theorem D with

$$
A_{1}(p)=\left(A_{2} / p\right)^{2 p} \text {. }
$$

To see how this result follows from the Theorem of Jenkins and Oikawa [4] we assume that $z_{0}=0, R=1$. Then the above authors proved that if $f(z)=\sum_{0}^{\infty} a_{n} z^{n}$ is $p$-valent in $|z|<1$, and $0<r<1$, we have

$$
M\left(r, f(z)-a_{0}\right) \leqq \mu_{p} A_{0}(p)(1-r)^{-2 p}
$$

where

$$
\mu_{p}=\max _{1 \leqq \nu \leqq p}\left|a_{\nu}\right|, A_{0}(p)=A_{0}^{p}
$$


Now Cauchy's inequality yields for $n>p$

$$
\left|a_{n}\right| \leqq A_{0}(p) \mu_{p} r^{-n}(1-r)^{-2 p},
$$

and choosing $r=1-p /(2 n)$, we deduce that

$$
\left|a_{n}\right| \leqq A_{0}^{p} \mu_{p}\left(\frac{2 n}{p}\right)^{2 p}\left(1-\frac{p}{2 n}\right)^{-n} \leqq A_{0}^{p} \mu_{p} 2^{3 p}(n / p)^{2 p} .
$$

This proves (1.11) with $A_{1}(p)=\left(8 A_{0} / p^{2}\right)^{p}$, when $R=1, z_{0}=0$. We deduce the general case of Theorem $\mathrm{D}$, by considering $f\left(z_{0}+R z\right)$ instead of $f(z)$.

Suppose now that $R \geqq 2$. Then (1.11) shows that for $j>p$

$$
\frac{\left|f^{(j)}\left(z_{0}\right)\right|}{j !} \leqq \max _{1 \leqq \nu \leqq p}\left(8 A_{0} j^{2} / p^{2}\right)^{p} R^{\nu-j} \frac{\left|f^{(\nu)}\left(z_{0}\right)\right|}{\nu !} .
$$

We write $F(z)=f^{\prime}(z)$, and deduce that for $R \geqq 2, n>p$

$$
\frac{\left|F^{(n-1)}\left(z_{0}\right)\right|}{(n-1) !} \leqq \max _{0 \leqq \nu p-1} \frac{\left|F^{(\nu)}\left(z_{0}\right)\right|}{\nu !}
$$

provided that

$$
\frac{\left|f^{(n)}\left(z_{0}\right)\right|}{(n-1) !} \leqq \max _{1 \leqq \nu ’ p} \frac{\left|f^{(\nu)}\left(z_{0}\right)\right|}{(\nu-1) !} .
$$

In view of (6.1) this condition is satisfied for $n>2 p$ if

$$
n\left(8 A_{0} n^{2} / p^{2}\right)^{p} 2^{-(1 / 2) n}<1,
$$

i.e. provided that $n \geqq A p$. Thus if the hypotheses of Theorem 1 hold with a fixed $R \geqq 2$, and $n \geqq A p$ then (6.2) holds for all $z_{0}$ and so $F(z)=f^{\prime}(z)$ has index at most $A p$. If $R<2$, then disks of radius 2 can be covered by almost $A R^{-2}$ disks of radius $R$, so that $f(z)$ is $p_{1}$-valent in disks of radius 2 , where $p_{1}<A p R^{-2}$. Thus we obtain the first statement of Theorem 1 also in this case.

Next we deduce similarly from (6.1) that $F(z)$ has index less than $p$, provided that (6.2) holds for $n>p$, i.e. provided that

$$
j\left(8 A_{0} j^{2} / p^{2}\right)^{p} R^{p-j}<1, \quad j>p .
$$

This is equivalent to

$$
(j-p) \log R>2 p\{(\log j / p)+A\}+\log j,
$$

which is satisfied provided that $\log R>A p$. This proves the second part of Theorem 1, and completes the proof of that Theorem.

6.1. We next prove Theorem 4. For this purpose we apply Theorem 3 to $F(z)=f(t z)-w$, where $w$ is any complex number, $f(z)$ is the 
function of Theorem 4 , and $t=C R /(p+1)$. Our hypotheses imply that

$$
\left(\frac{C R}{t}\right)^{p+1} \frac{F^{p+1}(z)}{(p+1) !} \leqq \max _{1 \leqq \nu \leqq p}\left(\frac{C R}{t}\right)^{\nu} \frac{\left|F^{(\nu)}(z)\right|}{\nu !}
$$

for $\left|t z-z_{0}\right| \leqq R$, i.e. $\left|z-t^{-1} z_{0}\right| \leqq t^{-1} R$, and since $C \leqq 1 / 2$, $t^{-1} R \geqq 2(p+1)$. Also (6.3) shows that

$$
\begin{aligned}
\left|F^{(p+1)}(z)\right| & \leqq \max _{1 \leqq \nu \leqq p}\left(\frac{t}{C R}\right)^{p+1-\nu} \frac{(p+1) !}{\nu !}\left|F^{(\nu)}(z)\right| \\
& \leqq \max _{1 \leqq \nu \leqq p}\left|F^{(\nu)}(z)\right| \leqq \max _{0 \leqq \nu \leqq p}\left|F^{(\nu)}(z)\right|
\end{aligned}
$$

since $C R=(p+1) t$. Thus $F(z)$ satisfies the hypothesis of Theorem 3 , with $t^{-1} R$ instead of $R$, and $n=p+1$, and we deduce that $F(z)$ has at most $p$ zeros in $\left|z-t^{-1} z_{0}\right| \leqq(p+1)^{1 / 2} / 12 \cdot 2$, so that the equation $f(z)=w$ has at most $p$ roots in

$$
\left|z-z_{0}\right|<t(p+1)^{1 / 2} / 12 \cdot 2=C R /\left\{12 \cdot 2(p+1)^{1 / 2}\right\},
$$

i.e. $f(z)$ is $p$-valent in this disk. This proves Theorem 4 .

We next prove the Corollary. Suppose first that $f(z)$ is an integral function, which has b.v.d., so that for some $R, f(z)$ assumes no value more than $p$ times in any disk of radius $R$. Then it follows from Theorem 1, that $f^{\prime}(z)$ has bounded index. Furthermore if $R \geqq A_{6}^{p}$, then the index $N$ of $f^{\prime}(z)$ is at most $p-1$. Suppose now that with $R=A_{6}^{N}$, where $N$ is the index of $f(z)$, we have $p(R) \leqq N$, so that $f(z)$ assumes no value more than $N$ times in any disk of radius $R$. Then Theorem 1 shows that $f^{\prime}(z)$ has index at most $N-1$, which gives a contradiction. This proves the second inequality of the Corollary.

Next if $f^{\prime}(z)$ has bounded index $N$, it follows that we have for all $z$

$$
\frac{\left|f^{(N+2)}(z)\right|}{(N+1) !} \leqq \max _{1 \leqq \nu \leqq N+1} \frac{\left|f^{(\nu)}(z)\right|}{(\nu-1) !}
$$

and hence à fortiori

$$
\frac{\left|f^{(N+2)}(z)\right|}{(N+2) !} \leqq \max _{1 \leqq \nu \leqq N+1} \frac{\left|f^{(\nu)}(z)\right|}{\nu !} .
$$

Thus we may apply Theorem 4 , with $p=N+1, C=1 / 2, R=2$, and deduce that $f(z)$ is $(N+1)$-valent in every disk of radius

$$
r=1 /\left\{12 \cdot 2(N+2)^{1 / 2}\right\} \text {. }
$$

Thus $f(z)$ has b.v.d. and $p(r) \leqq N+1$. This completes the proof of the Corollary of Theorem 4. 
6.2. It remains to prove Theorem 5. The first inequality in (2.6) follows at once from Theorem 1. The other inequality in (2.6) will follow at once from (2.7), which we now proceed to prove. The method is similar to that employed by Tijdeman [10]. We write

$$
f(z)=\sum_{0}^{\infty} a_{n} z^{n},
$$

and suppose, as we may do without loss of generality, that

$$
\max _{1 \leqq \nu \leqq N+1}\left|a_{\nu}\right|=1 \text {. }
$$

Thus

$$
\frac{\left|f^{(\nu+1)}(0)\right|}{(N+1)^{\nu}}=\frac{\left|a_{\nu+1}\right|(\nu+1) !}{(N+1)^{\nu}} \leqq 1, \quad 0 \leqq \nu \leqq N .
$$

Thus, applying (2.5) to $f^{\prime}(z)$, we obtain for $|z| \leqq R$

$$
\left|f^{\prime}(z)\right| \leqq e^{(N+1) R} \text {. }
$$

We deduce that for $|z|=R>0$

$$
\left|f(z)-a_{0}\right| \leqq \int_{0}^{R} e^{(N+1) t} d t<e^{(N+1) R} .
$$

We proceed to estimate the number $n(\rho)$ of zeros of $f(z)$ in $|z|<\rho$. Suppose first that

$$
\left|a_{0}\right|>e^{(N+1) \rho} .
$$

Then for $|z| \leqq \rho$

$$
|f(z)|>\left|a_{0}\right|-\left|f(z)-a_{0}\right|>e^{(N+1) \rho}-e^{(N+1) \rho}=0,
$$

so that is this case $n(\rho)=0$. Thus we may assume that

$$
\left|a_{0}\right| \leqq e^{(N+1) \rho}
$$

and we deduce that

$$
|f(z)| \leqq 2 e^{(N+1) R}, \quad R \geqq \rho .
$$

It now follows from the normalisation (6.4) and Cauchy's inequality that

$$
M(1, f) \geqq 1, \quad \text { where } \quad M(r, f)=\max _{|z|=r}|f(z)| .
$$

Thus there exists $z_{0}$, such that $\left|z_{0}\right|=1$, and $\mid f\left(z_{0}\right) \geqq 1$. We apply Jensen's inequality to

$$
\phi(z)=f\left(z_{0}+z\right)
$$


in the circle $|z| \leqq R$, and deduce that the number $n_{1}$, of zeros of $\phi(z)$ in $|z| \leqq \rho+1$, satisfies

$$
n_{1} \leqq \frac{\log \{M(R, \phi) /|\phi(0)|\}}{\log (R /(\rho+1))} \leqq \frac{(N+1)(R+1)+\log 2}{\log \{R /(\rho+1)\}},
$$

since

$$
M(R, \phi)=\max _{|z|=R}|\phi(z)| \leqq 2 e^{(N+1)(R+1)} .
$$

Choosing $R=e(\rho+1)$, we deduce that

$$
n(\rho) \leqq n_{1} \leqq(N+1)(e(\rho+1)+1)+\log 2<(N+1) e(\rho+2) .
$$

The same argument can be applied to $f(z)-w$, whose derivative is the same as that of $f(z)$ and we deduce that the equation $f(z)=w$ has at most $(N+1) e(\rho+2)$ roots in any disk of radius $\rho \geqq 1$. This proves (2.7). In particular setting $R=1$ we deduce the right hand inequality of (2.6) with $A_{8}=3 e$.

6.3. It remains to prove the Corollary. We first take $R_{1}=1$, and assume that $f^{\prime}(z)$ has index $N$. Then it follows from (2.6) that

$$
p(1) \geqq A_{7}(N+1) \text {. }
$$

On the other hand in view of (2.7) we have for $R_{2}>1$

$$
p\left(R_{2}\right) \leqq 3 e(N+1) R_{2} \leqq \frac{3 e}{A_{7}} R_{2} p(1),
$$

so that (2.8) holds if $R_{1}=1$. The general case now follows, since we may apply this result to $f\left(R_{1} z\right)$.

The result of the Corollary shows in particular that for any b.v.d. function $f(z), p(R)=O(R)$ as $R \rightarrow \infty$, and also by Theorem 2, Corollary $2, f^{\prime}(z)$ and so $f(z)$ has at most exponential type. These two result answer affirmatively a previous conjecture [3, problem 2.28]. In conclusion it is worth pointing out that since the index is rather easy to deal with we can obtain various applications for functions of bounded value distribution. One example of such an application is (2.8). Also if $f^{\prime}(z)$ has index $N, f(z)$ has index at most $N+1$, as we have already pointed out. Thus if $f(z)$ has b.v.d. the same is true of successive integrals of $f(z)$. The converse is false in general. However we can prove

THEOREM 8. If $f(z)$ is p-valent in $\left|z-z_{0}\right|<R$, then $f^{\prime}(z)$ has at most $p-1$ zeros in $\left|z-z_{0}\right|<A(p) R$. 
To see this we note that Theorem $\mathrm{D}$, with $j=p+1$, $A_{1}(p)=\left(A_{2} / p\right)^{2 p}$, shows that for $\left|z-z_{0}\right| \leqq(1 / 2) R$

$$
\left|f^{(p+1)}(z)\right| \leqq(p+1) !\left(A_{2}(p+1) / p\right)^{2 p} \max _{1 \leqq \nu \leqq p} \frac{\left(\frac{1}{2} R\right)^{\nu-(p+1)}\left|f^{(\nu)}(z)\right|}{\nu !} .
$$

If $R=R_{p}=2(p+1) !\left\{A_{2}(p+1) / p\right\}^{2 p}<A_{10}^{p}$, this yields

$$
\left|f^{(p+1)}(z)\right| \leqq \max _{1 \leqq \nu \gg p}\left|f^{(\nu)}(z)\right|,\left|z-z_{0}\right| \leqq \frac{1}{2} R .
$$

It now follows from Theorem 3 , that $f^{\prime}(z)$ has at most $p-1$ zeros in $\left|z-z_{0}\right|<p^{1 / 2} / 12 \cdot 2$. Thus we deduce the desired conclusion with $A(p)=A_{11}^{p}$, where $A_{11}$ is a small absolute constant. If $R \neq R_{p}$, we obtain our conclusion by considering $f\left(R_{p} z / R\right)$ instead of $f(z)$.

6.4. We complete the paper by giving an example of a function $f(z)$ such that $f(z)$ has bounded index but $f^{\prime}(z)$ does not.

Let

$$
F(z)=e^{z} \prod_{n=1}^{\infty}\left(1+\frac{z}{2^{n}}\right)^{n} .
$$

Then $F(z) e^{-z}$ is an integral function of order zero with zeros of arbitrarily high order. Thus $F(z)$ cannot have bounded index. Also

$$
F(z)=O\left\{e^{-C|z|}\right\} \text { and so } F^{\prime}(z) \longrightarrow 0, \text { as }|z| \longrightarrow \infty,
$$

for $\pi / 2+\delta \leqq \arg z \leqq 3 \pi / 2-\delta$, where $C$ is a positive constant depending on $\delta$.

Next

$$
\frac{F^{\prime}(z)}{F(z)}=1+\sum_{n=1}^{\infty} \frac{n}{z+2^{n}} \longrightarrow 1, \text { as }|z| \longrightarrow \infty, \text { for }|\arg z| \leqq \pi-\delta .
$$

We now set

$$
f(z)=B+\int_{0}^{z} F(z) d z .
$$

It follows from (6.6) that by a suitable choice of $B$ we can make sure that

$$
|f(z)| \geqq 1, \text { for } \frac{3 \pi}{4} \leqq \mid \arg z \leqq \frac{5 \pi}{4}
$$

so that

$$
\left|F^{\prime \prime}(z)\right| \leqq A|f(z)|
$$


in this range in view of (6.6). Also for $|\arg z| \leqq 3 \pi / 4$, we have

$$
\left|F^{\prime}(z)\right| \leqq A|F(z)|
$$

in view of (6.7). Thus we have in the whole plane

$$
\left|f^{\prime \prime}(z)\right| \leqq A \max \left\{|f(z),| f^{\prime}(z) \mid\right\}
$$

where $A$ is a suitable constant, and so by Theorem 4

$$
\int f(z) d z
$$

has b.v.d. in the plane and so by the Corollary of Theorem $4 f(z)$ has bounded index, and in fact if $\varepsilon$ is a sufficiently small positive number $f(\varepsilon z)$ has index one. However the derivatives of $f(\varepsilon z)$ do not have bounded index.

I am grateful to the referee for a number of minor corrections and for having drawn my attention to Shah [9], where the author gives an example different from mine of a function with bounded index, whose derivatives do not have bounded index.

\section{REFERENCES}

1. M. Biernacki, Sur les fonctions multivalentes d'ordre p. C. R. Acad. Sci., (Paris) 203 (1936), 449-451.

2. S. Dancs and P. Turán, On the distribution of values of a class of entire functions, Publ. Math. Debrecen, 11 (1964), 257-272.

3. W. K. Hayman, Research problems in function theory, (Athlone press of the University of London 1967).

4. J. A. Jenkins and K. Oikawa, On results of Ahlfors and Hayman, Illinois J. Math., 15 (1971), 664-671.

5. W. J. Kim, The Schwarzian derivative and multivalence, Pacific J. Math., 31 (1969), $717-724$.

6. B. Lepson, Differential equations of infinite order, hyper Dirichlet series and entire functions of bounded index, Lecture notes, 1966, Summer institute on Entire Functions, University of California, La Jolla.

7. S. M. Shah, Entire functions of bounded index, Proc. Amer. Math. Soc., 19 (1968), 1017-1022.

8. —_, Entire functions satisfying a linear differential equation, J. Math. and Mech., 18 (1968), 131-136.

9. - On entire functions of bounded index whose derivatives are of unbounded index, J. London Math. Soc., (2) 4 (1971), 127-139.

10. R. Tijdeman, On the distribution of the values of certain functions, Thesis (Amsterdam 1969).

11. On the number of zeros of general exponential polynomials, Nederl. Akad. Wetensch. Proc. Ser. A 74 = Indag. Math. 33 (1971), 1-7.

12. P. Turán, Eine neue Methode in der Analysis und deren Anwendungen, (Akademiai Kiado, Budapest, 1953). 
13. H. Wittich, Zur Kennzeichung linearer Differentialgleichungen mit konstanten Koeffizienten; Festband zum, 70. Geburtstag von R. Nevanlinna, Springer Berlin 1966, 128-134.

Received August 27, 1971.

IMPERIAL COLLEGE

LONDON S.W. 7. 
